\title{
Estradiol suppresses neuronal firing activity and c-Fos expression in the lateral habenula
}

\author{
CHUN-YING LI ${ }^{1}$, MEI-YING SONG ${ }^{2}$, MIN HUANG ${ }^{1}$, JI-CHENG LI $^{1}$, JIN-YU XIAO $^{1}$ and HUA ZHAO ${ }^{1}$ \\ ${ }^{1}$ Department of Physiology, College of Basic Medical Sciences, Jilin University; \\ ${ }^{2}$ Neuroscience Research Center, First Hospital of Jilin University, Changchun, Jilin 130021, P.R. China
}

Received July 16, 2014; Accepted April 28, 2015

DOI: $10.3892 / \mathrm{mmr} .2015 .3942$

\begin{abstract}
Estradiol is an ovarian steroid hormone that regulates physiological functions in the central nervous system, including mood, cognition, sleep and mental state. Emerging evidence has revealed that there is an enrichment of cells that express the estrogen receptor in the lateral habenula $(\mathrm{LHb})$ region, however, the precise biological functions of estradiol on neurons in the LHb remain to be elucidated. The present study aimed to determine the effects of estradiol on $\mathrm{LHb}$ neurons, by observing neuronal firing activity, and c-Fos mRNA and protein expression levels in the $\mathrm{LHb}$ using whole cell recording, reverse transcription-quantitative polymerase chain reaction and western blotting. Ovariectomized female Wistar rats were supplemented with a range of estradiol doses across five groups: Ovariectomized (no treatment); Oil (sesame oil); low estradiol; medial estradiol (ME) and high estradiol. Circulating serum estradiol levels were assessed over a 33 day period following surgery. Estradiol suppressed spontaneous firing activity in $\mathrm{LHb}$ neurons $(\mathrm{P}<0.05$, compared with firing rates prior to estradiol treatment), which suggested a role for this hormone in regulating neuronal activity. Estradiol replacement therapy resulted in sustained serum estradiol levels for $\sim 3$ weeks after surgery. The mRNA and protein levels of c-Fos were significantly downregulated in $\mathrm{LHb}$ tissues from $\mathrm{ME}$ rats as compared with Oil animals $(\mathrm{P}<0.05)$. In conclusion, the present study demonstrated that estradiol suppresses neuronal activities in the $\mathrm{LHb}$ region, suggesting that the $\mathrm{LHb}$ may be a potential target for estradiol action.
\end{abstract}

Correspondence to: Professor Hua Zhao, Department of Physiology, College of Basic Medical Sciences, Jilin University, 126 Xinmin Street, Changchun, Jilin 130021, P.R. China

E-mail: zhua@jlu.edu.cn

Key words: estrogen, lateral habenula, ovariectomized, c-Fos, spontaneous action

\section{Introduction}

The lateral habenula (LHb) region of the brain, has a critical role in controlling numerous behaviorally important functions, including reward, emotional regulation, pain, stress, sleep and cognition (1-3). The LHb is comprised of multiple subnuclei and it is considered an integral neuronal component for transmitting information between the limbic forebrain and the midbrain regions. The LHb inhibits the activity of neurons that contain monoaminergic neurotransmitters, including dopamine and serotonin, via efferent projections that terminate in the substantia nigra pars compacta, ventral tegmental area and raphe nuclei (2-6). Dysfunction of the $\mathrm{LHb}$ is associated with a number of neurological disorders, including sleep disturbance, depression, drug addiction and schizophrenia (1,7-11).

A number of studies have demonstrated that neurons in the LHb express high levels of estrogen receptor (ER)- $\alpha$ and ER- $\beta$, which preferentially localizes to nuclei, axon terminals and postsynaptic dendrites (12-15). It has also been suggested that estrogen signaling in the LHb has an essential role in regulating female reproductive behaviors during pregnancy and post-parturition (14). Together, these findings support a role for gonadal hormones in the LHb. Nevertheless, the sensitivity of neurons in the LHb to hormonal regulation remains poorly understood.

In the present study, the effects of estradiol signaling on the LHb were investigated using a model of estradiol replacement therapy in ovariectomized rats. The electrophysiological responses of LHb neurons to estradiol, the most biologically potent member of the estrogen family, were evaluated.

\section{Materials and methods}

Animals. A total of 70 female Wistar rats aged 9-10 weeks, were obtained from the Laboratory Animal Center of Jilin University (Changchun, China; certification no. SCXK(Ji)2007-0003). Animals were housed in standard temperature-, humidity- and light-controlled conditions (temperature $22 \pm 1^{\circ} \mathrm{C}$; humidity $40-50 \%$; lighting, $12 \mathrm{~h}$ light/dark cycle). All animals were housed in the facility under these conditions for 1 week prior to experiments. All experiments with animals were approved by the Animal Ethical Committee of Jilin University and performed in 
accordance with Decree No. 2 of the State and Technology Commission of China (Approved by the State Council on October 31, 1988, and promulgated by the State Science and Technology Commission on November 14, 1988).

Surgery and estradiol replacement treatments. On day 0 , bilateral ovariectomy of rats was performed as previously described (16), with minor modifications. Silastic ${ }^{\circledR}$ capsules (Dow Corning, Midland, MI, USA) containing either 0, 90, 180 or $360 \mu \mathrm{g} / \mathrm{ml}$ of $17 \beta$-estradiol (Sigma-Aldrich, St. Louis, MO, USA) in a solution with sesame oil (Sigma-Aldrich) were implanted subcutaneously for the Oil, low estradiol (LE), medial estradiol (ME) and high estradiol (HE) groups, respectively. The Silastic ${ }^{\circledR}$ capsules were sealed with Type A biogum (Dow Corning) and preincubated in sesame oil for $16 \mathrm{~h}$. Following surgery, animals received daily intraperitoneal injections of 40,000 units of penicillin for 3 days.

Evaluation of serum estradiol content. Blood (1 ml) was collected from the tail vein at 3, 5, 7, 9, 11, 18, 26 and 33 days after surgery $(17,18)$. The samples were incubated for $1 \mathrm{~h}$ at room temperature and centrifuged at $3,764 \mathrm{x} g$ for $20 \mathrm{~min}$. The serum was separated and stored at $4{ }^{\circ} \mathrm{C}$ for up to $24 \mathrm{~h}$ prior to serum estradiol quantification by chemiluminescence using a UniCel DxI 800 Immunoassay System (Beckman Coulter, Brea, CA, USA).

Electrophysiology. Rats were anesthetized by intraperitoneal injection of $10 \%$ chloral hydrate. The rats were then sacrificed by decapitation and then $\mathrm{LHb}$ tissues were extracted for sample preparation. Tissue sections containing the LHb regions were dissected and $350 \mu \mathrm{m}$ coronal slices were prepared. Slices were immediately incubated in oxygenated artificial cerebrospinal fluid (ACSF) (19) containing $135 \mathrm{mM} \mathrm{NaCl}, 3.5 \mathrm{mM}$ $\mathrm{KCl}, 26 \mathrm{mM} \mathrm{NaHCO}, 1.25 \mathrm{mM} \mathrm{NaH}_{2} \mathrm{PO}_{4}, 10 \mathrm{mM}$ glucose, $2.5 \mathrm{mM} \mathrm{CaCl}_{2}$ and $1.2 \mathrm{mM} \mathrm{MgSO}_{4}$ for $25 \mathrm{~min}$ at $34^{\circ} \mathrm{C}$ and incubated at room temperature $\left(20-25^{\circ} \mathrm{C}\right)$ for $1 \mathrm{~h}$ prior to patch clamp analysis. For the duration of the patch clamp analysis experiments, samples were perfused with oxygenated ACSF (2-3 $\mathrm{ml} / \mathrm{min})$ at $20-25^{\circ} \mathrm{C}$ and cells were continuously monitored using a BX51WI Olympus microscope (Olympus, Tokyo, Japan). Patch microelectrodes were guided stereotactically and pulled to a tip resistance of 3-5 M $\Omega$ on a PP-83 micropipette puller (Narishige, Tokyo, Japan) and filled with internal solution containing $125 \mathrm{mM} \mathrm{K}$-gluconate, $20 \mathrm{mM} \mathrm{KCl,} 10 \mathrm{mM}$ HEPES, 5 mM EGTA, $4 \mathrm{mM}$ Mg-ATP, $0.4 \mathrm{mM} \mathrm{NaGTP} \mathrm{and}$ $0.1 \mathrm{mM} \mathrm{CaCl}{ }_{2}$. Estradiol $(1 \mu \mathrm{M})$ dissolved in dimethyl sulfoxide (DMSO) were administered into the superfusion fluid within $5 \mathrm{~min}$.

Signals were amplified using an EPC10 patch clamp amplifier and digitized at $10 \mathrm{kHZ}$ (HEKA Elektronik Dr. Schulze GmbH, Pfalz, Germany) and data were collected using PatchMaster v2x32 (HEKA Elektronik Dr. Schulze $\mathrm{GmbH}$ ) software and analyzed with FitMaster v2x65 (HEKA Elektronik Dr. Schulze GmbH).

Immunohistochemical analysis. Animals were anesthetized by intraperitoneal injection of $20 \%$ urethane $(6 \mathrm{ml} / \mathrm{kg}$ body weight). The hearts of the animals were flushed with phosphate-buffered saline (PBS) and perfused with a solution of 4\% paraformaldehyde (PFA) in 0.1 M PBS (pH 7.2-7.4). Brain tissue slices which contained $\mathrm{LHb}$ were fixed in $4 \%$ PFA for $24 \mathrm{~h}$, dehydrated in a series of ethanol and embedded in paraffin. Paraffin-embedded tissues were sectioned into $2 \mu \mathrm{m}$ thick coronal slices, c-Fos protein was detected using a rabbit anti-rat primary antibody specific for c-Fos-specific antibody (1:500 dilution; cat. no. ab102699; Abcam, Cambridge, England). Immunostaining was detected using an UltraSensitive $^{\mathrm{TM}}$ SP kit according to the manufacture's instructions (KIT-9710; Fuzhou Maxim Biotech Inc., Fujian, China). Briefly, samples were labeled with a biotin-labeled secondary antibody (solution C, $50 \mu \mathrm{l}$ ) and streptavidin-anti-biotin-peroxidase (solution D, $50 \mu \mathrm{l}$ ). Immunostaining was visualized using 3,3'-diaminobenzidine tetrahydrochloride kit (DAB-0031; Fuzhou Maxim Biotech Inc.). Samples were counterstained with hematoxylin, washed with running tap water, dehydrated in a series of ethanol, cleared in xylene and mounted with neutral balsam.

Sections were subsequently counterstained with hematoxylin prior to visualization on a BX53 microscope (Olympus, Tokyo, Japan). The mean optical density of c-Fos staining in LHb sections was determined by analysis of randomly selected fields and quantification with Image-Pro Plus 6.0 software (Media Cybernetics, Silver Spring, MD, USA).

\section{Reverse transcription-quantitative polymerase chain reaction} $(R T-q P C R)$. Total RNA was isolated from the tumor tissues using TRIzol reagent according to the manufacturer's instructions (Invitrogen Life Technologies, Carlsbad, CA, USA). First-strand cDNA was synthesized by reverse transcription of $2 \mu \mathrm{g}$ total RNA using an RNA PCR kit (Takara Bio., Inc., Shiga, Japan). Primer sequences used for RT-qPCR reactions were as follows: c-fos, forward 5'-AGCATGGGCTCCCCTGTCA-3' and reverse 5'-GAGACCAGAGTGGGCTGCA-3'; GAPDH, forward 5'-GGGTGATGCTGGTGCTGAGTATGT-3' and reverse 5'-AAGAATGGGAGTTGCTGTTGAAGT-3'.

Reactions were run in an ABI PRISM 7900 HT thermocycler (Applied Biosystems, Foster City, CA, USA) for 35 cycles. The relative mRNA expression of the target gene was normalized to that of GAPDH. Data were analyzed from three independent experiments (three animals).

Western blot analysis. The LHb tissue samples were lysed in RIPA solution for $30 \mathrm{~min}$ on ice. Lysates were centrifuged at $12,000 \mathrm{x} \mathrm{g}$ for $10 \mathrm{~min}$ at $4^{\circ} \mathrm{C}$ and the supernatants were collected and stored at $-80^{\circ} \mathrm{C}$. Protein concentrations for each sample were determined using a Bradford assay according to the manufacturer's instructions (Nanjing Jiancheng Biotechnology Institute, Nanjing, China). Protein (40 $\mu \mathrm{g}$ ) from each sample was separated in an 8 or $12 \%$ sodium dodecyl sulfate polyacrylamide gel and transferred onto a polyvinylidene difluoride membrane (Millipore, Billerica, MA, USA) at $4^{\circ} \mathrm{C}$. Proteins were detected with a rabbit anti-rat c-Fos-specific antibody (1:1,000 dilution; cat. no. ab102699; Abcam, Cambridge, UK), horseradish peroxidase-conjugated goat anti-rabbit IgG secondary antibodies (1:6,000 dilution; ZDR-5118; Beijing Zhongshan Golden Bridge Biotechnology Co., Ltd., Beijing, China) and visualized using enhanced chemiluminescence solution and X-ray films. Band densities were determined by Scion version 4.02 imaging software (Scion Corporation, Frederick, MD, USA). 


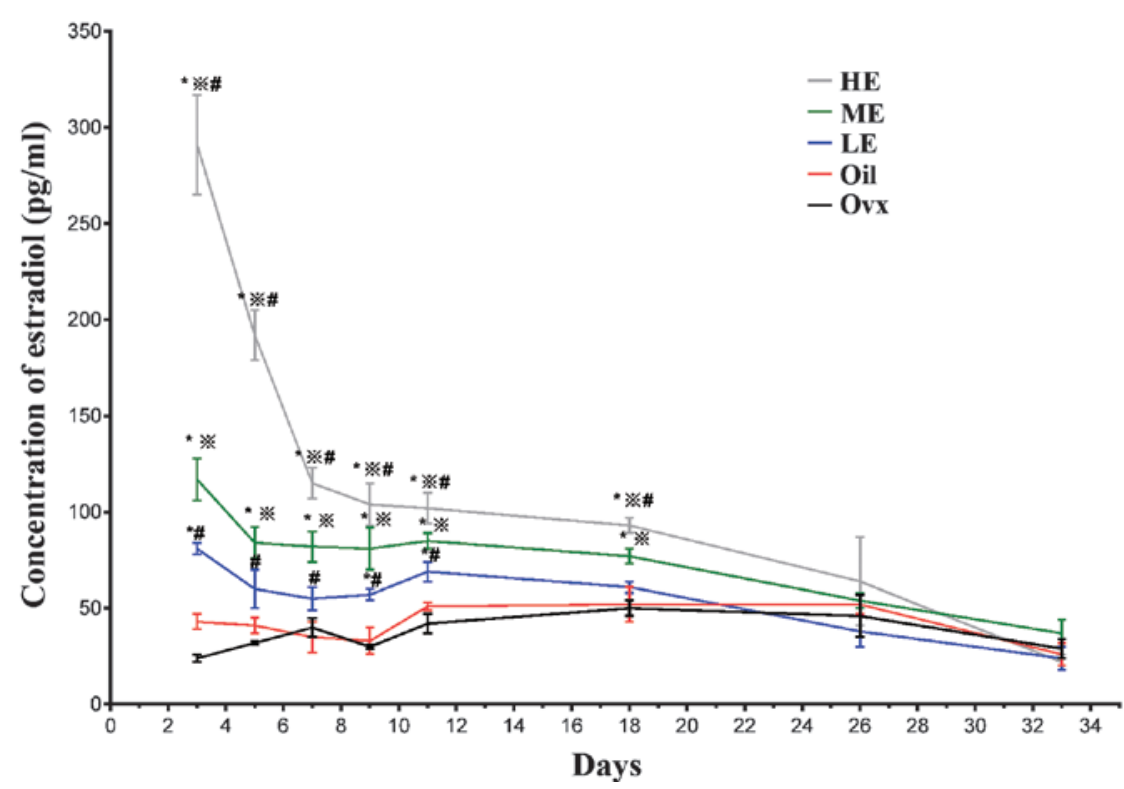

Figure 1. Serum concentrations of estradiol in ovariectomized rats with estradiol hormone replacement therapy. $\mathrm{n}=6$ animals in each group. * $\mathrm{P}<0.05$, compared with the Oil group; ${ }^{*} \mathrm{P}<0.05$, compared with the LE group; ${ }^{~} \mathrm{P}<0.05$, compared with the ME group. LE, low estradiol; ME, medial estradiol; HE, high estradiol; Ovx, ovariectomized.

Statistical analysis. Data were analyzed by SPSS 11.0 software (SPSS, Inc., Chicago, IL, USA) and are presented as the mean \pm standard error of the mean. Statistical significance was determined using one-way analysis of variance and two-tailed t-test and displayed using GraphPad Prism 6 software (GraphPad Software, Inc., San Diego, CA, USA).

\section{Results}

Estradiol replacement therapy is sustained for up to 3 weeks in ovariectomized rats. No significant differences were found in the serum estradiol levels of untreated ovariectomized rats when compared with those in the Oil group ( $P>0.05$; Fig. 1), which demonstrated that the sesame oil carrier solution had no biological effects in this system. Serum estradiol levels in the medial estradiol (ME) and high estradiol (HE) groups were significantly elevated at $3,5,7,9,11$ and 18 days post-surgery compared with the Oil group ( $\mathrm{P}<0.05 ;$ Fig. 1). Animals in the low estradiol (LE) dosage group exhibited increased serum estradiol levels only at 9 and 11 days post-surgery $(\mathrm{P}<0.05$; Fig. 1). However, no statistically significant differences were found in serum estradiol levels for any group compared with the controls at 26 and 33 days post-surgery ( $\mathrm{P}>0.05$; Fig. 1 ). These findings demonstrated that hormone replacement therapy in ovariectomized rats was effective for up to 3 weeks. Given that animals in the ME group appeared to maintain serum estradiol levels for the longest period, these animals were used to investigate the effects of estrogen hormones on c-Fos expression in the LHb neurons.

Estradiol suppresses spontaneous firing activity in LHb neurons. The effects of estradiol (dissolved in DMSO) on the functional activity of neurons in the LHb derived from normal female rats were examined using patch clamp electrophysiology techniques. Administration of a $0.01-0.05 \%$ DMSO solution to LHb tissue samples demonstrated no effect on the spontaneous firing rate of neurons in the LHb area. However, exposure of $\mathrm{LHb}$ tissue samples to $1 \mu \mathrm{M}$ estradiol, resulted in a $40.89 \%$ decrease in the average firing rate of the neurons from $4.33 \pm 0.90 \mathrm{~Hz}$ prior to treatment with estradiol to $2.65 \pm 0.63 \mathrm{~Hz}$ following administration of estradiol ( $\mathrm{P}<0.01$; Fig. 2A-C). When the estradiol-treated samples were washed for $5 \mathrm{~min}$ with ACSF, the firing rate was recovered to baseline levels (Fig. 2A and B). Together these results suggest that estradiol inhibits the spontaneous firing activities of neurons in the LHb region and that this effect is reversible when estrogen is attenuated.

Expression of c-Fos in the LHb of ME-treated rats. The proto-oncogene c-Fos is a widely accepted marker of neuron activation within the central nervous system. To understand the effect of estradiol on LHb neurons, the expression of c-Fos was examined at the mRNA and protein levels in ME-treated and control animals. The mRNA expression of c-Fos was significantly decreased in ME-treated animals compared with that of the control animals 7 days post-surgery ( $\mathrm{P}<0.05$; Fig. $3 \mathrm{~A})$. These results were supported by similar findings at the protein level that demonstrated reduced quantities of c-Fos protein in ME-derived LHb tissues compared with the equivalent control tissues $(\mathrm{P}<0.05$; Fig. 3B and $\mathrm{C})$.

Immunohistochemical analysis of tissue sections stained for c-Fos revealed that the intensity of staining as well as the number of c-Fos-positive neurons was decreased in the LHb region of ME rats compared with Oil animals $(\mathrm{P}<0.05$; Fig. 4A and $\mathrm{B})$.

\section{Discussion}

The $\mathrm{LHb}$ region is known to regulate a wide range of biological functions, including reward and aversion (1), and dysfunction in LHb neurons is associated with numerous neurological disorders, including sleep disturbance and depression (9). Estradiol regulates numerous physiological functions in 
A

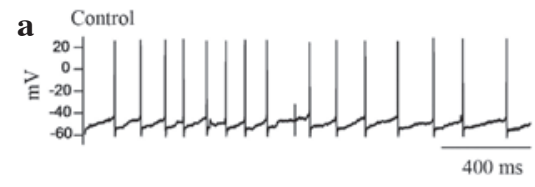

B

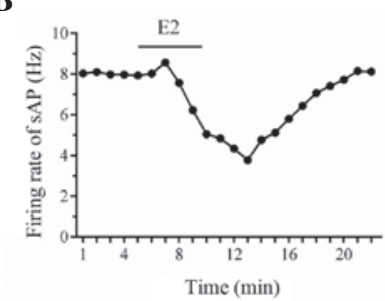

b Perfusing $1 \mu \mathrm{M}$ estradiol for $5 \mathrm{~min}$

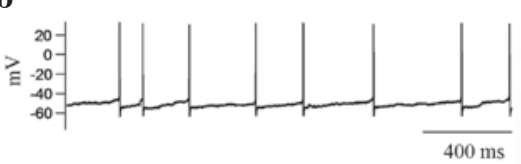

c After $5 \mathrm{~min}$ of washing with ACSF

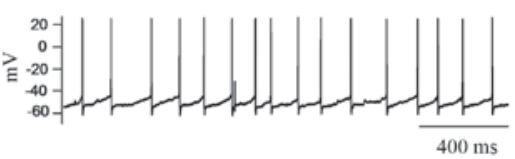

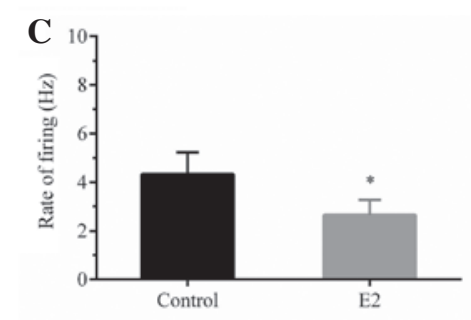

Figure 2. Estradiol inhibits electrophysiological activity in LHb neurons. (A) Representative data of the sAP (Aa) prior to, (Ab) during and (Ac) following $1 \mu \mathrm{M}$ estradiol perfusion. (B) Time course data of the effect of $1 \mu \mathrm{M}$ estradiol on the firing rate of the sAP. (C) Altered firing rate of LHb neurons in response to estradiol perfusion. " $\mathrm{P}<0.05$, compared with the control $(\mathrm{n}=9)$. LHb, lateral habenula; sAP, spontaneous action potential.

A

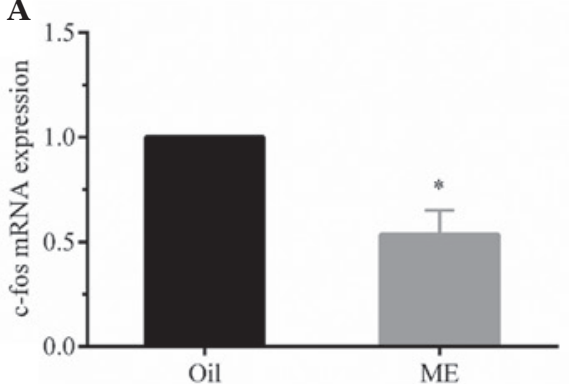

B

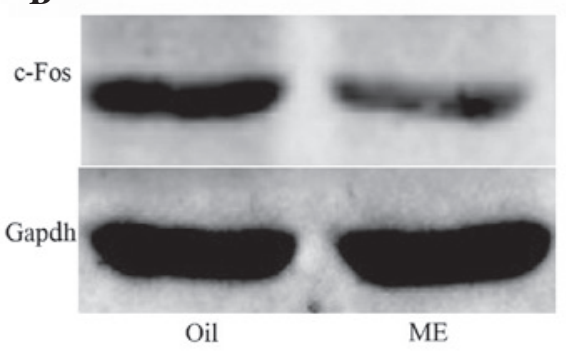

C

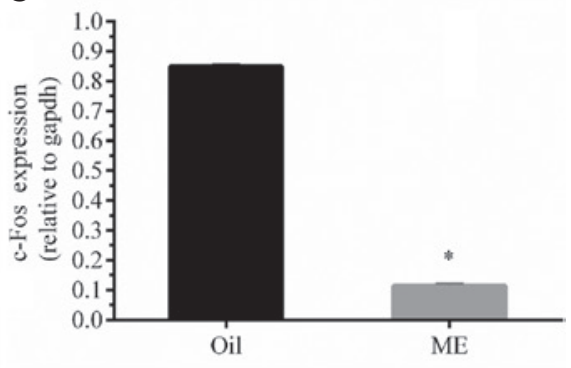

Figure 3. Estradiol downregulates the mRNA and protein expression of c-Fos in LHb tissues. Animals from the control Oil group and the ME group were compared at 7 days post-surgery. The c-Fos expression was determined by (A) mRNA and (B and C) protein quantification. "P<0.05, compared with the Oil group. LHb, lateral habenula; ME, medial estradiol.

A
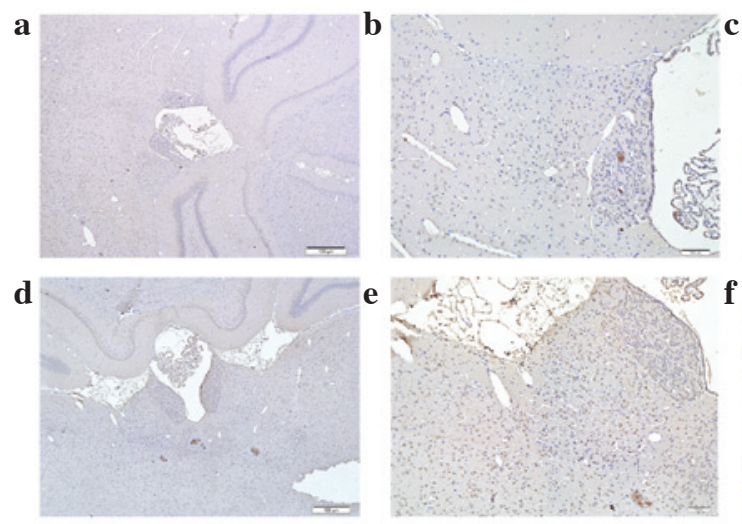
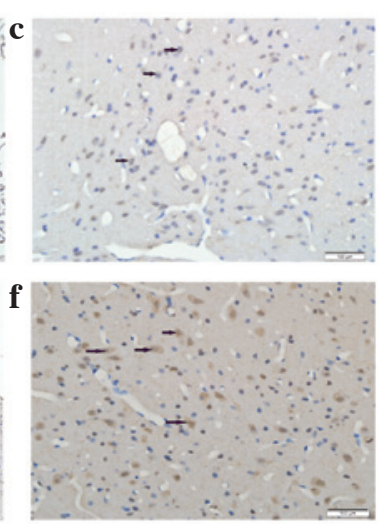

$\mathbf{B}$

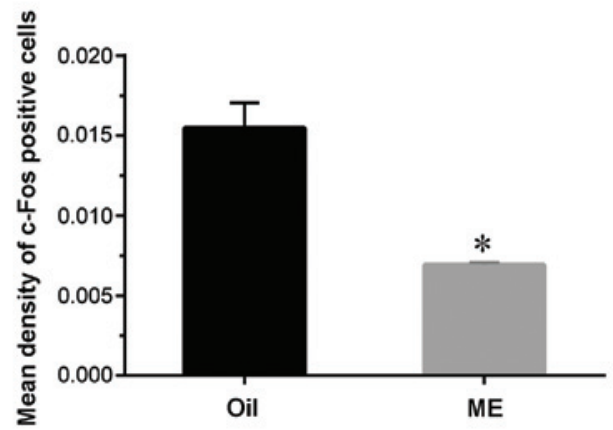

Figure 4. c-Fos immunohistochemical staining in the LHb after 7 days of estradiol hormone replacement therapy. (A) Representative immunostaining. (Aa and Ad) magnification, x40; (Ab and Ae) magnification, x200; (Ac and Af) magnification, x400. (a-c) Estradiol treated group; (d-f) Oil group. Black arrows indicate c-Fos immunoreactive cells. (B) Mean density of c-Fos positive cells was quantified. " $\mathrm{P}<0.05$, compared with the Oil group. LHb, lateral habenula. 
the central nervous system, and changes in the levels of estrogen are associated with the pathogenesis of psychiatric illness (20-22). The present study investigated the association between estrogen hormones and neuronal activity in the $\mathrm{LHb}$ region and demonstrated that this hormone functions to inhibit neuron activity in the $\mathrm{LHb}$ region.

The ovariectomized female rat has been widely used as an ideal rodent model to investigate the effect of hormone replacement on pain responses and analgesia $(23,24)$. To understand the effects of estradiol on neuronal activities in the LHb area, estradiol replacement therapy was applied in ovariectomized female rats to avoid the effects of endogenous hormones on the outcome. It was found that subcutaneous implantation of Silastic ${ }^{\circledR}$ capsules containing estradiol provided sustained serum hormone levels for $\sim 3$ weeks after implantation. In addition, no significant differences were found in serum estradiol levels between animals in the Oil group and in the ovariectomized group receiving no treatment, indicating that the sesame carrier solution did not affect the results.

Patch clamp recording analysis demonstrated that exogenous estradiol inhibited spontaneous firing activities in $\mathrm{LHb}$ neurons, which suggested a role for this hormone in negative regulation in the LHb. The proto-oncogene c-Fos is a marker of activated neurons and is typically transiently induced in response to multiple extracellular stimuli $(25,26)$. The present study demonstrated that estradiol replacement therapy resulted in a significant decrease in c-Fos expression in $\mathrm{LHb}$ tissues.

Our results suggest that estradiol may suppress neuronal firing activity and c-Fos expression in the LHb, however, additional investigations are required to clarify the precise mechanisms underlying its action on LHb neurons.

In conclusion, the present study demonstrated that estradiol hormone inhibits neuronal activity in the LHb region. These results may provide a rationale for the development of estradiol pharmacological interventions for neurological disorders, via effects on the LHb neuronal activity.

\section{Acknowledgements}

This study was supported by the Bethune Medical Scientific Research Support Plan (grant no. 2013205029) and Beihua University doctoral research fund (Miss Chun-ying Li).

\section{References}

1. Zhao H, Zhang BL, Yang SJ and Rusak B: The role of lateral habenula-dorsal raphe nucleus circuits in higher brain functions and psychiatric illness. Behav Brain Res 277: 89-98, 2015.

2. Christoph GR, Leonzio RJ and Wilcox KS: Stimulation of the lateral habenula inhibits dopamine-containing neurons in the substantia nigra and ventral tegmental area of the rat. J Neurosci 6: 613-619, 1986.

3. Hikosaka O: The habenula: From stress evasion to value-based decision-making. Nat Rev Neurosci 11: 503-513, 2010.
4. Wang RY and Aghajanian GK: Physiological evidence for habenula as major link between forebrain and midbrain raphe. Science 197: 89-91, 1977.

5. Matsumoto M and Hikosaka O: Lateral habenula as a source of negative reward signals in dopamine neurons. Nature 447: 1111-1115, 2007.

6. Bernard R and Veh RW: Individual neurons in the rat lateral habenular complex project mostly to the dopaminergic ventral tegmental area or to the serotonergic raphe nuclei. J Comp Neurol 520: 2545-2558, 2012.

7. Ellison G: Stimulant-induced psychosis, the dopamine theory of schizophrenia and the habenula. Brain Res Brain Res Rev 19: 223-239, 1994.

8. Lecourtier L, Neijt HC and Kelly PH: Habenula lesions cause impaired cognitive performance in rats: Implications for schizophrenia. Eur J Neurosci 19: 2551-2560, 2004.

9. Aizawa H, Cui W, Tanaka K and Okamoto H: Hyperactivation of the habenula as a link between depression and sleep disturbance. Front Hum Neurosci 7: 826, 2013.

10. Sartorius A, Kiening KL, Kirsch P, et al: Remission of major depression under deep brain stimulation of the lateral habenula in a therapy-refractory patient. Biol Psychiatry 67: e9-e11, 2010.

11. Li B, Piriz J, Mirrione M, et al: Synaptic potentiation onto habenula neurons in the learned helplessness model of depression. Nature 470: 535-539, 2011.

12. Shughrue PJ, Lane MV and Merchenthaler I: Comparative distribution of estrogen receptor-alpha and -beta mRNA in the rat central nervous system. J Comp Neurol 388: 507-525, 1997.

13. Laflamme N, Nappi RE, Drolet G, Labrie C and Rivest S: Expression and neuropeptidergic characterization of estrogen receptors (ERalpha and ERbeta) throughout the rat brain: Anatomical evidence of distinct roles of each subtype. J Neurobiol 36: 357-378, 1998.

14. Wagner CK, Silverman AJ and Morrell JI: Evidence for estrogen receptor in cell nuclei and axon terminals within the lateral habenula of the rat: Regulation during pregnancy. J Comp Neurol 392: 330-342, 1998.

15. Milner TA, Thompson LI, Wang G, et al: Distribution of estrogen receptor beta containing cells in the brains of bacterial artificial chromosome transgenic mice. Brain Res 1351: 74-96, 2010.

16. Deurveilher S, Rusak B and Semba K: Estradiol and progesterone modulate spontaneous sleep patterns and recovery from sleep deprivation in ovariectomized rats. Sleep 32: 865-877, 2009.

17. Frank P, Schoenhard GL and Burton E: A method for rapid and frequent blood collection from the rat tail vein. J Pharmacol Methods 26: 233-238, 1991.

18. Staszyk C, Bohnet W, Gasse H and Hackbarth H: Blood vessels of the rat tail: A histological re-examination with respect to blood vessel puncture methods. Lab Anim 37: 121-125, 2003.

19. Sun J, Chu Z and Moenter SM: Diurnal in vivo and rapid in vitro effects of estradiol on voltage-gated calcium channels in gonadotropin-releasing hormone neurons. J Neurosci 30: 3912-3923, 2010.

20. Gutiérrez-Lobos K, Scherer M, Anderer P and Katschnig H: The influence of age on the female/male ratio of treated incidence rates in depression. BMC Psychiatry 2: 3, 2002.

21. Li R and Shen Y: Estrogen and brain: Synthesis, function and diseases. Front Biosci 10: 257-267, 2005.

22. Soares CN: Mood disorders in midlife women: Understanding the critical window and its clinical implications. Menopause 21: 198-206, 2014.

23. Fillingim RB and Ness TJ: Sex-related hormonal influences on pain and analgesic responses. Neurosci Biobehav Rev 24: 485-501, 2000.

24. Mannino CA, South SM, Quinones-Jenab V and Inturrisi CE: Estradiol replacement in ovariectomized rats is antihyperalgesic in the formalin test. J Pain 8: 334-342, 2007.

25. Harris JA: Using c-fos as a neural marker of pain. Brain Res Bull 45: 1-8, 1998.

26. Kovács KJ: Measurement of immediate-early gene activation - c-fos and beyond. J Neuroendocrinol 20: 665-672, 2008 